\title{
Case Report: A Case of Endocarditis and Embolic Stroke in a Child, Suggestive of Acute Q Fever Infection
}

\author{
Maria Bitsori, ${ }^{1}$ Eleni Vergadi, ${ }^{1}$ loannis Germanakis, ${ }^{1}$ Maria Raissaki, ${ }^{2}$ and Emmanouil Galanakis ${ }^{1 *}$ \\ ${ }^{1}$ Department of Paediatrics, Heraklion University Hospital, Medical School, University of Crete, Heraklion, Greece; ${ }^{2}$ Department of Radiology, \\ Heraklion University Hospital, Medical School, University of Crete, Heraklion, Greece
}

\begin{abstract}
Acute $\mathrm{Q}$ fever is usually asymptomatic or is associated with a mild self-limited course and a favorable outcome. The occurrence of endocarditis during acute infection by Coxiella burnetii is an emerging clinical entity observed in adults that has been attributed to an autoimmune complication of early infection. Herein, we report the first case of a previously healthy 2-year-old child with endocarditis complicated by septic embolic stroke, in which the identified microbiological evidence was suggestive of acute rather than chronic $C$. burnetii infection. The development of endocarditis in this case occurred in the absence of any autoimmune reaction, but in the context of a very mild form of congenital heart disease, a small ventricular septal defect, which might serve as a predisposing factor for endocarditis. This case suggests that acute $Q$ fever endocarditis may affect children as well and can be attributed not only to autoimmune mechanisms but also to a potential effect of the infectious agent per se on the cardiac endothelium in patients with underlying heart defects, regardless of their severity.
\end{abstract}

\section{INTRODUCTION}

Q fever is a globally distributed zoonosis caused by the fastidious intracellular bacterium Coxiella burnetii. ${ }^{1,2}$ In humans, infection from $C$. burnetii may be either acute or chronic. ${ }^{1,3,4}$ Acute $Q$ fever in children is usually a self-limited febrile infection with nonspecific symptoms that has been rarely associated with adverse outcomes. ${ }^{1,5,6}$ However, information on acute $Q$ fever complications in children is limited because many cases usually surpass unnoticed.

Endocarditis is regarded a severe, almost exclusively chronic complication of $Q$ fever in immunocompromised patients or those with underlying valvular defects. ${ }^{7}$ Acute $Q$ fever endocarditis, that is, endocarditis during the course of a primary acute $C$. burnetii infection, has been recently recognized in adults and has mainly been attributed to autoimmune reaction to infection..$^{1,2,5,7,8}$ In this article, we report the first case of a child with an insignificant congenital heart disease that developed endocarditis complicated by embolic stroke, with serology suggestive of primary $C$. burnetii rather than chronic infection.

\section{CASE PRESENTATION}

A 2-year-old girl presented with a 3-day history of highgrade fever $\left(39.7^{\circ} \mathrm{C}\right)$, vomiting, and lethargy. Her past medical history was unremarkable apart from the presence of a nonsignificant small ventricular septal defect (VSD).

On presentation, she was confused, disoriented, and pale. Her vital signs were body temperature $39.2^{\circ} \mathrm{C}$, blood pressure $95 / 52 \mathrm{mmHg}$, pulse rate 154 beats/minute, respiratory rate 30 breaths/minute, peripheral oxygen saturation $98 \%$ in room air, and capillary refill time 3 seconds. Physical examination was unremarkable apart from a holosystolic heart murmur $(3 / 6)$ at the left third intercostal space. Soon after admission, she developed focal clonic seizures on her left side and rapid progression to coma, requiring seizure control and admission in the pediatric intensive care unit.

* Address correspondence to Emmanouil Galanakis, Department of Paediatrics, University of Crete, Voutes, Heraklion 71110, Greece. E-mail: emmgalan@uoc.gr
Initial laboratory examination revealed leukopenia (white blood cells $2,400 / \mathrm{mm}^{3}$, neutrophils $1,746 / \mathrm{mm}^{3}$, and lymphocytes $528 / \mathrm{mm}^{3}$ ) without substantial elevation of erythrocyte sedimentation rate $(31 \mathrm{~mm} /$ hour $)$ and C-reactive protein $(0.2$ $\mathrm{mg} / \mathrm{dL})$. Aspartate aminotransferase was $81 \mathrm{U} / \mathrm{L}$ (5-40 U/L), but the remaining serum and urine biochemistry was normal. Analysis of cerebrospinal fluid revealed no cells, normal protein and glucose levels, and a negative PCR for bacteria (Neisseria meningitis, Streptococcus pneumoniae, Haemophilus influenzae, Listeria monocytogenes, Escherichia coli, Pseudomonas aeruginosa, Streptococcus spp., and Staphylococcus aureus) and viruses (herpes simplex virus 1,2 [HSV1/ 2], Epstein-Barr virus [EBV], cytomegalovirus [CMV], enteroviruses, and human herpesvirus-6 [HHV-6]). Tuberculin skin test and serology for viruses (IgM and IgG titers for EBV, CMV, adenovirus [ADV], and enteroviruses), Toxoplasma gondii, and Mycoplasma pneumoniae were negative.

Brain imaging with computed tomography on admission showed two areas of subcortical white matter hypodensity without abnormal enhancement on the right cerebral hemisphere, suggestive of edema. On day 3 of hospitalization, brain magnetic resonance imaging showed multiple, hyperintense foci at the supratentorial vascular right carotid territory (Figure 1A). There was extensive finger-like vasogenic subcortical edema, surrounding these foci (Figure 1B). These findings were suggestive of septic embolic lesions. On the same day, a transthoracic echocardiogram revealed the presence of a previously known small $(4 \mathrm{~mm})$ restrictive VSD (pressure gradient $100 \mathrm{mmHg}$ ) that was located just beneath both aortic (AO) and pulmonary valves (PV) (doubly committed type) (Figure 2A). Thickening of the $A O$ valve leaflets was also noted without identification of vegetations (Figure 2B). Repeated transthoracic echocardiography 5 days later revealed the emergence of mild AO insufficiency as a new finding (Figure 2C).

The clinical presentation pointed to the diagnosis of infectious endocarditis as the case fulfilled one major (new valvular regurgitation) and three minor (emboli, fever, and predisposing condition for endocarditis) diagnostic criteria. ${ }^{9}$ However, blood cultures did not yield any microorganism, and further examinations toward the identification of potential 

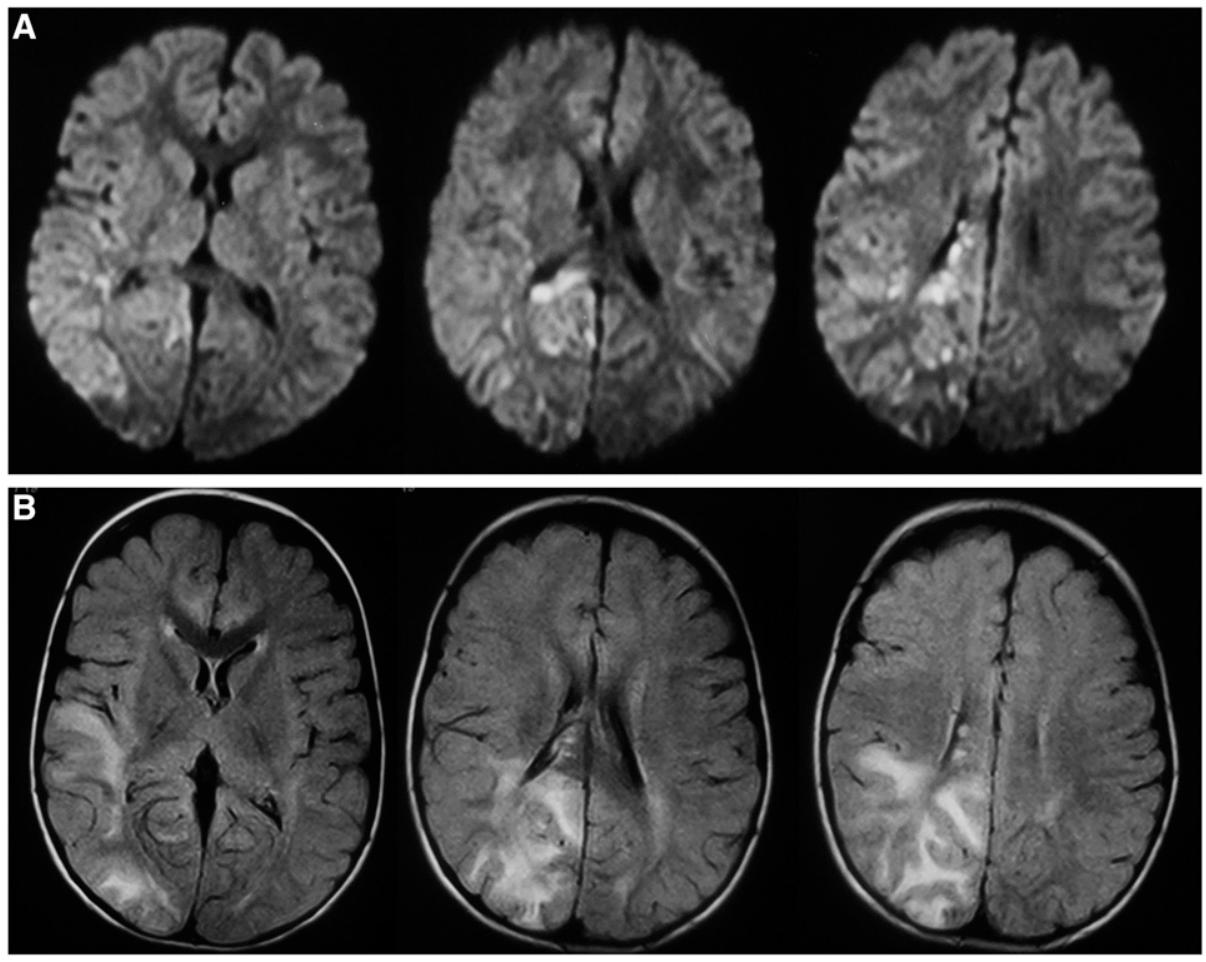

FiguRE 1. (A) Axial diffusion images at three different levels show numerous hyperintense foci at a supratentorial vascular right carotid (middle cerebral artery and posterior cerebral artery) territory. (B) Axial T2-w sequences at the same three different levels as in (A). There is extensive fingerlike vasogenic subcortical edema, surrounding these foci at the subcortical white matter of the right parietal lobe including the cingulate gyrus.

causative agents were performed. ${ }^{10}$ The autoantibody screen, including antinuclear antibodies, rheumatoid factor, and antiphospholipid antibodies such as lupus anticoagulant, anticardiolipin antibodies and anti- $\beta 2$ glycoprotein I antibodies, measured by direct ELISA, were negative. Cultures were negative for Haemophilus spp., Aggregatibacter spp., Cardiobacterium hominis, Eikenella corrodens, and Kingella spp. organisms. Indirect immunofluorescence assays (IFAs) for other fastidious slow-growing bacteria, such as Bartonella spp. and Rickettsiae spp., ELISA for Chlamydia pneumoniae and Brucella spp., and blood PCR for Tropheryma whipplei were negative. The serological results regarding $C$. burnetti (based on IFAs) revealed a substantial increase in phase II antibodies (Table 1). At this point (day 20 of hospitalization), blood samples were sent to a reference unit (Laboratory of Clinical Bacteriology, Parasitology, and Zoonoses, Medical School, the University of Crete) for confirmatory testing with culture and blood PCR amplification (using a pair of primers that target the gene IS1111 transposase elements) ${ }^{11}$ for C. burnetii, but the results were negative. The titers of IgG and IgM against phase I of $C$. burnetii antibody test remained low throughout the course of disease in our patient (Table 1).

Detailed epidemiological history revealed that the child had no history of contact with farm animals or consumption of unpasteurized milk, but there were cattle on neighboring properties. The patient lives is an urban area, endemic for
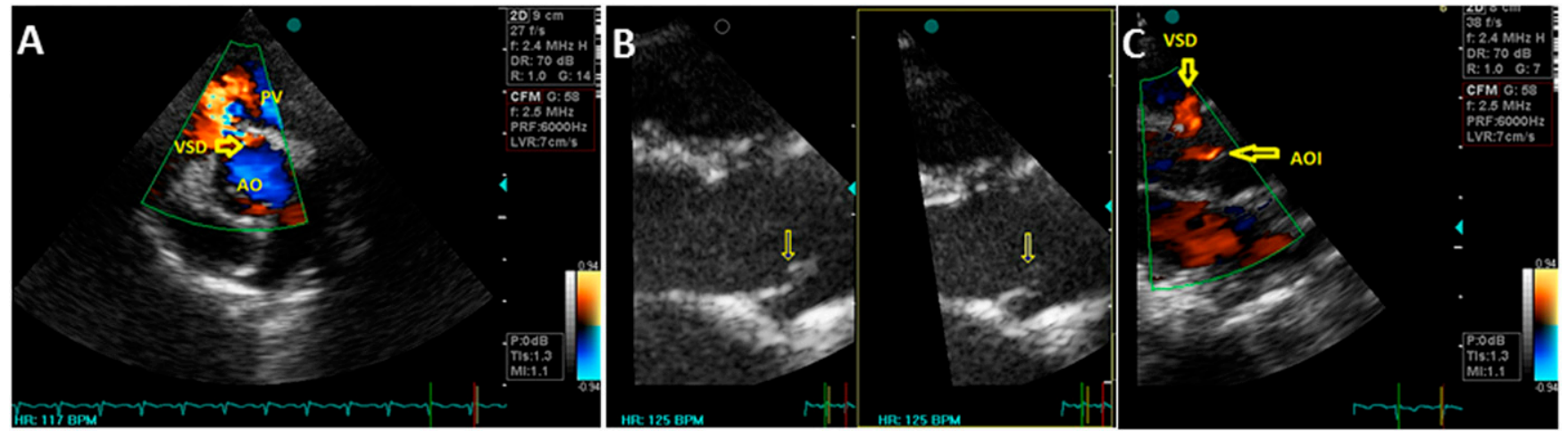

FIGURE 2. (A) Color Doppler imaging, parasternal short axis echocardiographic view at the level of great arteries origin. Increased velocity blood flow (left to right shunt) through a restrictive double-committed ventricular septal defect (VSD-arrow) located underneath the aortic (AO) and pulmonary valves. (B) 2D imaging, parasternal long axis echocardiographic view, $A O$ root with $A O$ valve leaflets in the systole. Thickening of $A O$ valve leaflet tips (arrow). (C) Color Doppler imaging, parasternal long axis echocardiographic view, AO root with AO valve regurgitation (aortic insufficiency [AOI]-arrow). The left to right shunt through the subaortic (VSD-arrow) is also visible. This figure appears in color at www.ajtmh.org. 
TABLE 1

Antibody titers against of $C$. burnetii

\begin{tabular}{|c|c|c|c|c|}
\hline \multirow[b]{3}{*}{ Day/month after the onset of symptoms } & \multicolumn{2}{|c|}{ C. burnetii } & \multicolumn{2}{|c|}{ C. burnetii } \\
\hline & \multicolumn{2}{|c|}{ Phase II antibodies } & \multicolumn{2}{|c|}{ Phase I antibodies } \\
\hline & $\lg M$ & $\lg G$ & $\lg M$ & $\lg G$ \\
\hline 5th day & $1 / 25$ & $1 / 60$ & $1 / 25$ & Negative \\
\hline 21st day & $1 / 800$ & $1 / 120$ & $1 / 50$ & Negative \\
\hline 28th day & $1 / 1,600$ & $1 / 480$ & $1 / 200$ & $1 / 60$ \\
\hline 45th day & $1 / 800$ & $1 / 480$ & $1 / 100$ & $1 / 120$ \\
\hline 3 months & $1 / 100$ & $1 / 60$ & $1 / 100$ & $1 / 60$ \\
\hline 5 months & Negative & $1 / 20$ & Negative & $1 / 60$ \\
\hline 12 months & Negative & Negative & Negative & $1 / 20$ \\
\hline
\end{tabular}

C. burnetii $=$ Coxiella burnetii.

C. burnetii, and her symptoms appeared during the spring, when lambing usually occurs and the environment can be easily contaminated from the birth by-products of infected animals. $^{12}$

After the positive serology had become available, the initial empiric treatment for infectious endocarditis was changed to cotrimoxazole and rifampicin for 3 months, and then continued with clarithromycin and rifampicin for a total of 18 months. The fever subsided 3 days later, and the laboratory abnormalities returned to normal within 1 week. A 4-fold decrease in C. burnetii phase II titers was noted 5 months after treatment initiation, and a 5-year follow-up period had been uneventful without any adverse effects or relapses. Coxiella burnetii antiphase II and antiphase I antibodies were evaluated every 6 months for the first 12 months of follow-up and then annually for the following 5 years, and no increase in antibody titers was noted.

\section{DISCUSSION AND REVIEW OF THE LITERATURE}

We report a 2-year-old child presenting with an unusually severe form of acute $Q$ fever, including endocarditis and embolic stroke. Most cases of acute $C$. burnetii infection are asymptomatic, and severe complications such as prolonged fever, pneumonia, hepatitis, myocarditis, pericarditis, or encephalitis have been only rarely reported. ${ }^{1,5,6,13}$

Coxiella burnetii is often an etiologic agent of culturenegative endocarditis. However, most $C$. burnetii-associated endocarditis cases develop late, usually 2 months to 1 year following acute $Q$ fever. ${ }^{14-16}$ Recently, the clinical entity of acute $Q$ fever endocarditis has been described in adults who did not fulfill the usual chronic $Q$ fever endocarditis criteria. ${ }^{17}$ This new clinical entity has been attributed to an autoimmune complication of early infection associated with antiphospholipid syndrome and specifically with elevation of anticardiolipin antibodies. ${ }^{2,8,16}$

To the best of our knowledge, this is the first report of acute $Q$ fever endocarditis in a child. Our patient did not have high levels of $C$. burnetii antiphase I IgG titers, and, for this reason, she did not fulfill the classic modified Duke's criteria for chronic $Q$ fever endocarditis. ${ }^{9}$ However, the only microbiological evidence that we identified in this case was that of an active, acute $C$. burnetii infection signified by the elevation of antiphase II antibody titers. Serological evidence of active infection with organism consistent with infectious endocarditis $^{9}$ is listed among the minor criteria in the modified Duke's criteria for the diagnosis of infectious endocarditis.
Thus, it appears that this child developed acute $Q$ fever endocarditis similarly to the entity that has been already described in adults. ${ }^{8}$ We should point out, however, that the lack of additional evidence of $C$. burnetii infection, such as positive PCR or later development of phase I antibodies, does not allow a firmly established diagnosis of acute $Q$ fever endocarditis but rather points to a highly suggestive case of acute C. burnetii infection. In our case also, there was no elevation of anticardiolipin antibodies or any other indices of autoimmune process; thus, the rapid development of endocarditis may be attributed to the infectious agent per se. The child did not have a significant risk factor for infectious endocarditis such as a valvular defect. ${ }^{18}$ However, her specific doubly committed type of VSD, with exposure of both $A O$ and PV valves to turbulent blood flow, might have facilitated bacteria colonization. Indeed, development of subacute bacterial endocarditis has been described in children with unrepaired VSDs of this type. $^{19}$

Serological diagnosis of acute $Q$ fever requires phase II C. burnetii lgM titers greater than 1/50 and/or IgG titers greater than 1/200, whereas the diagnosis of chronic $Q$ fever requires clinical presentation lasting for more than 6 months and a high phase I antibody titer of $1 / 800$ or greater. ${ }^{4}$ However, in endemic areas, such as our area which is endemic for C. burnetii, a higher cutoff value has been suggested for the diagnosis. ${ }^{20}$ For example, based on the local seroprevalence, a cutoff value of $1 / 240$ has been suggested for phase II IgG titers. ${ }^{20}$ Because of delays in clinical suspicion and difficulties with organism culture, serology remains the main diagnostic tool for $Q$ fever. ${ }^{1,21}$ In our patient, we attempted PCR in a blood sample, collected on the day 20 of hospitalization, which was negative; however, PCR in blood specimens is usually negative 23 weeks after acute infection. ${ }^{3,21,22}$

The management of $Q$ fever endocarditis is challenging in children. Based on in vitro susceptibility studies, tetracyclines, rifampicin, and cotrimoxazole are bactericidal against C. burnetii. ${ }^{23}$ The combination of doxycycline and hydroxychloroquine has been proven to be the most efficacious and is currently recommended for the treatment of $Q$ fever endocarditis in adults ${ }^{22,24}$ and children older than 8 years. ${ }^{3,22}$ Many experts recommend doxycycline in severe cases of $Q$ fever, regardless of age. ${ }^{1,6,15,22}$ However, prolonged treatment with doxycycline and hydroxychloroquine bears the risk of dental staining and retinopathy in young children, and alternative treatment with cotrimoxazole is recommended by the CDC for pregnant women and younger ages. ${ }^{3,22}$ The minimum duration of treatment in native valve $Q$ fever endocarditis is 
18 months, and a 4-fold decrease in IgG titers is a useful indicator of treatment success. ${ }^{6,15,21,24}$ In this 2-year-old toddler, we treated alternatively with cotrimoxazole and rifampicin for 3 months, and then with clarithromycin and rifampicin for 18 months. Treatment with doxycycline was considered but was not eventually necessary because of her excellent clinical response. Heart valve insufficiency did not evolve, and the child remained healthy over the subsequent 5 years, without any impairment or relapse.

\section{CONCLUSION}

Infectious endocarditis during the course of acute $Q$ fever may be encountered in children and may be attributed not only to autoimmune complications but also to the direct effect of the infectious agent per se in patients with vulnerable cardiac endothelium. Acute $Q$ fever infection may pose a risk of rapid development of endocarditis in children with even minor and common forms of congenital heart abnormalities, such as VSDs.

Received December 15, 2019. Accepted for publication July 1, 2020.

Published online August 3, 2020.

Authors' addresses: Maria Bitsori, Department of Pediatrics, Heraklion University Hospital, Crete, Greece, E-mail: bitmar2@gmail.com. Eleni Vergadi, loannis Germanakis, and Emmanouil Galanakis, Department of Pediatrics, School of Medicine, University of Crete, Greece, E-mails: eleni.vergadi@med.uoc.gr, yannis.germanakis@ gmail.com, and emmgalan@uoc.gr. Maria Raissaki, Department of Radiology, School of Medicine, University of Crete, Greece, E-mail: mraissaki@yahoo.gr.

\section{REFERENCES}

1. Maltezou HC, Raoult D, 2002. Q fever in children. Lancet Infect Dis 2: 686-691.

2. Melenotte $C$ et al., 2018. Clinical features and complications of Coxiella burnetii infections from the French national reference center for Q fever. JAMA Netw Open 1: e181580.

3. Anderson $A$ et al., 2013. Diagnosis and management of $Q$ fever-United States, 2013: recommendations from $C D C$ and the $Q$ fever working group. MMWR Recomm Rep 62: 1-30.

4. Scola BL, 2002. Current laboratory diagnosis of $Q$ fever. Semin Pediatr Infect Dis 13: 257-262.

5. Maltezou HC, Constantopoulou I, Kallergi C, Vlahou V, Georgakopoulos D, Kafetzis DA, Raoult D, 2004. Q fever in children in Greece. Am J Trop Med Hyg 70: 540-544.

6. Baquero-Artigao F, del Castillo F, Tellez A, 2002. Acute $Q$ fever pericarditis followed by chronic hepatitis in a two-year-old girl. Pediatr Infect Dis J 21: 705-707.
7. Raoult $D, 2012$. Chronic $Q$ fever: expert opinion versus literature analysis and consensus. $J$ Infect 65: 102-108.

8. Million $\mathrm{M}$ et al., 2016. Antiphospholipid antibody syndrome with valvular vegetations in acute Q fever. Clin Infect Dis 62: 537-544.

9. Baltimore RS et al., 2015. Infective endocarditis in childhood: 2015 update: a scientific statement from the American Heart Association. Circulation 132: 1487-1515.

10. Fournier PE et al., 2010. Comprehensive diagnostic strategy for blood culture-negative endocarditis: a prospective study of 819 new cases. Clin Infect Dis 51: 131-140.

11. Denison AM, Thompson HA, Massung RF, 2007. IS1111 insertion sequences of Coxiella burnetii: characterization and use for repetitive element PCR-based differentiation of Coxiella burnetii isolates. BMC Microbiol 7: 91.

12. Tselentis $Y$, Gikas A, Kofteridis D, Kyriakakis E, Lydataki N, Bouros D, Tsaparas N, 1995. Q fever in the Greek Island of Crete: epidemiologic, clinical, and therapeutic data from 98 cases. Clin Infect Dis 20: 1311-1316.

13. Ravid S, Shahar E, Genizi J, Schahor Y, Kassis I, 2008. Acute Q fever in children presenting with encephalitis. Pediatr Neurol 38: 44-46.

14. Al-Hajjar S, Hussain Qadri SM, al-Sabban E, Jäger C, 1997. Coxiella burnetii endocarditis in a child. Pediatr Infect Dis J 16 : 911-913.

15. Million M, Thuny F, Richet H, Raoult D, 2010. Long-term outcome of $Q$ fever endocarditis: a 26-year personal survey. Lancet Infect Dis 10: 527-535.

16. Million M, Walter G, Thuny F, Habib G, Raoult D, 2013. Evolution from acute $Q$ fever to endocarditis is associated with underlying valvulopathy and age and can be prevented by prolonged antibiotic treatment. Clin Infect Dis 57: 836-844.

17. Li JS, Sexton DJ, Mick N, Nettles R, Fowler VG, Jr., Ryan T, Bashore T, Corey GR, 2000. Proposed modifications to the duke criteria for the diagnosis of infective endocarditis. Clin Infect Dis 30: 633-638.

18. Habib G et al., 2015. 2015 ESC guidelines for the management of infective endocarditis. Eur Heart J 36: 3075-3128.

19. Gabriels C, De Backer J, Pasquet A, Paelinck BP, Morissens M, Helsen F, Van De Bruaene A, Budts W, 2017. Long-term outcome of patients with perimembranous ventricular septal defect: results from the Belgian registry on adult congenital heart disease. Cardiology 136: 147-155.

20. Vranakis I, Kokkini S, Chochlakis D, Sandalakis V, Pasparaki E, Minadakis G, Gikas A, Tselentis Y, Psaroulaki A, 2012. Serological survey of $Q$ fever in Crete, southern Greece. Comp Immunol Microbiol Infect Dis 35: 123-127.

21. Fournier PE, Marrie TJ, RaoultD, 1998. Diagnosis of Q fever. JClin Microbiol 36: 1823-1834.

22. Kersh GJ, 2013. Antimicrobial therapies for $Q$ fever. Expert Rev Anti Infect Ther 11: 1207-1214.

23. Yeaman MR, Mitscher LA, Baca OG, 1987. In vitro susceptibility of Coxiella burnetii to antibiotics, including several quinolones. Antimicrob Agents Chemother 31: 1079-1084.

24. Raoult D, Houpikian P, Tissot Dupont H, Riss JM, Arditi-Djiane J, Brouqui $P, 1999$. Treatment of $Q$ fever endocarditis: comparison of 2 regimens containing doxycycline and ofloxacin or hydroxychloroquine. Arch Intern Med 159: 167-173. 